\title{
EFFECTS OF SOLUTE-SOLVENT INTERACTIONS ON RADIATIONLESS DECAY OF THIOKETONES EXCITED TO THEIR $S_{2}$ - AND $T_{1}$-STATES
}

\author{
M. Szymański, M. Balicki, M. Binkowski, J. Kubicki, A. MaCiejeWski ${ }^{a}$,
} E. PaWlowska and T. Wróżowa

Institute of Physics and Faculty of Chemistry ${ }^{a}$

A. Mickiewicz University, Grunwaldzka 6, 60-780 Poznań, Poland

(Received August 21, 1995; revised version January 24, 1996)

Spectral and photophysical properties of a few aromatic thioketones in their $S_{2}$ - and $T_{1}$-states, in particular those determined by their interactions with solvents, are discussed. The reasons for a drastically different behaviour of thioketones in interactions with benzene as well as saturated hydrocarbons and perfluoroalkanes are analysed in more detail. Results of the time-resolved transient absorption measurements in the pico- and nanosecond time scale are given. An analysis of these results proves that a product of the decay of the $S_{2}$-state of xanthione in benzene is a new transient $\left(\tau_{1 / \mathrm{e}} \geq 60 \mathrm{ps}\right)$ individuum which, regarding the system properties and the conditions of the experiment, has been identified as an exciplex in $S_{2}$-state formed as a result of an efficient interaction with benzene molecule. Also in xanthione/alkane systems the involvement of a transient individuum was proved. Most probably, this individuum was a thioketyl radical which could be formed by hydrogen abstraction from a hydrocarbon molecule by xanthione in the $S_{2}$-state. Such individua act as intermediates in passing excitation to the triplet states of thioketones and are effective channels of the $S_{2}$-state decay. The changes observed in the transient absorption spectra of xanthione in $\mathrm{C}_{6} \mathrm{H}_{6}$ in the time range of $10^{-7}-10^{-6} \mathrm{~s}$ can be attributed to the formation of an excimer in the $T_{1}$-state as a result of the $T_{1}$-state selfquenching.

PACS numbers: 33.50.Dq, 33.50.Hv, 35.80.Be

\section{Introduction}

Thioketones, a group of compounds of unusually interesting spectral and photophysical properties, have been the subject of many publications and have not ceased to interest researchers in the unexpected effects following from their intramolecular properties as well as intra- and intermolecular interactions, particularly in solutions. A characteristic feature distinguishing the whole group of thioketones is strong fluorescence from the $S_{2}$-state occurring with violation of the 
classical Kasha rule [1]. For certain aromatic thioketones in perfluoroalkanes the quantum yield of this fluorescence reaches $\phi_{F} \approx 10^{-1}$ which is the highest value ever measured in solution for fluorescence from higher energy states [2].

Many spectral and photophysical properties of thioketones (thiones) predestine them to be model systems useful in many fields of fundamental research in physics and chemistry. Absorption spectra of thiocarbonyls are even richer than those of the corresponding carbonyls and more accessible in experiment thanks to the fact that the bands ascribed to the transitions to $T_{1}, S_{1}, S_{2}, S_{\mathbf{3}}$ and higher singlet states are better separated and appear in the visible and UV range. A weak absorption band of the molar extinction coefficient, $\varepsilon_{\mathrm{S}} \approx 10-30 \mathrm{M}^{-1} \mathrm{~cm}^{-1}$, corresponding to the orbitally forbidden transition to $S_{1}\left(n, \pi^{*}\right)$ is usually found in the red range. The band corresponding to the strong allowed transition to $S_{2}\left(\pi, \pi^{*}\right)$, of typical $\varepsilon$ values from $\approx 9000 \mathrm{M}^{-1} \mathrm{~cm}^{-1}$ to $c a .17000 \mathrm{M}^{-1} \mathrm{~cm}^{-1}$, appears in the blue or UV range. A large energy gap $\Delta E\left(S_{2}-S_{1}\right)$ and poor $S_{2}-S_{1}$ vibrational overlap are, on the one hand, responsible for a slow rate of $S_{2}$-state radiationless decay and, on the other hand, for pronounced $S_{2} \rightarrow S_{0}$ fluorescence [2, 3], which is moreover favoured by a high value of radiative rate constant $k_{\mathrm{F}}$.

Because of a strong spin-orbit coupling in thioketone molecules, the ban on combination of terms of different multiplicity is weakened and the absorption spectra of certain thiocarbonyls reveal a weak $\left(\varepsilon_{\mathrm{T}} \approx 1 \div 10 \mathrm{M}^{-1} \mathrm{~cm}^{-1}\right)$ but measurable and frequently well-resolved band of absorption to the $T_{1}$-state of primarily ${ }^{3}\left(n, \pi^{*}\right)$ character in whose vicinity there is a ${ }^{3}\left(\pi, \pi^{*}\right)$ state identified in most systems as $T_{2}$. A consequence of the enhanced probability of $T_{1} \leftarrow S_{0}$ transition is a strong phosphorescence characteristic of the majority of thiocarbonyls $[4,5]$, observed already at room temperature (RT) in solutions. For pyranthione in perfluoro-1,3-dimethylcyclohexane the $T_{1} \rightarrow S_{0}$ phosphorescence was the highest ever measured (at room temperature and in fluid solution) of the quantum yield $\phi_{\mathrm{P}}=0.47[6]$.

Thioketone molecules in excited states reveal other unusual features, although typical of these systems, determining their photophysical behaviour. One of them is very efficient quenching of the excited states, mainly the triplet ones but also the long-lived $S_{2}$-state, by the thione molecules in the ground state, the so-called selfquenching [7-10], which has serious consequences [11]. Another specific property of thioketones (thiones) is their unusually high reactivity in the excited states, mainly $S_{2}$ but $T_{1}$ as well, stemming from the properties of the thio group $(\mathrm{C}=\mathrm{S})[7,10]$ and long lifetimes of these states. In all solvents except perfluoroalkanes, thioketones excited to the $S_{2}$-state undergo very efficient deactivation in intermolecular processes which is manifested by a very significant shortening of the decay time and a decrease in the quantum yield of fluorescence. This drastic solvent effect can be explained assuming that interaction of excited thiones with solvents leads to formation of some transient individua. As indicated by measurements of the quantum yield of net photochemical consumption $[4,5,10,12]$ and an analysis of quantum yield of phosphorescence determined upon selective excitation $[13,14]$, these individua deactivate almost quantitatively to the thione and the solvent molecules in the ground state and the deactivation runs mostly with the involvement of $T_{1}$-state. Without the assumption about formation of these 
individua, no correct interpretation of photophysical properties, results of photochemical studies $[7,9,10]$ and studies on the quenching process $[8,10]$ would be possible. It is assumed that in the solvents sufficiently strongly interacting (e.g. $\mathrm{C}_{6} \mathrm{H}_{6}$ ) and simultaneously chemically inert $[7,10]$ the processes taking place between the thione in the $S_{2}$-state and the solvent molecules lead to formation of exciplexes (in this case exciplexes in the $S_{2}$-state) while in the chemically reactive solvents $[7,10,15,16]$ in which the interactions can be even very weak (e.g. alkanes) the transient individua are radicals. Despite many attempts, emission studies have so far failed to provide either direct evidence of existence of such individua or indirectly determine their properties $[7,10,17]$. Moreover, a few attempts have been made to use transient absorption spectroscopy with picosecond resolution to study the $S_{2}$-thione decay and to search for the transient individua. However, the obtained signals of transient absorption were either ascribed only to the thione molecules [18], no interpretation of the signal was given [19] or no signal was recorded [20].

The aim of our studies was to determine the properties of selected aromatic thioketones in the $S_{2}$-state in the solvents of essentially different but well-defined nature of interaction with thioketones in the $S_{2}$ and $T_{1}$-state. Moreover, this paper reports results of the application of pico- and nanosecond transient absorption spectroscopy to study the nature of interaction of excited $S_{2}$-thiones with benzene and saturated hydrocarbon molecules.

\section{Instrumentation and experimental procedures}

Transient absorption spectra of xanthione in the picosecond time scale were taken in the system shown schematically in Fig. 1. A source of picosecond light pulses of a $10 \mathrm{~Hz}$ repetition rate was a YAG: $\mathrm{Nd}^{3+}$ laser, YG 571C type (Quantel), equipped with generators of the second, third and fourth harmonic. The sample was excited with a $355 \mathrm{~nm}$ beam (third harmonic) of the energy in a single pulse up to $2 \mathrm{~mJ}$ at the pulse width of $12 \mathrm{ps}$ (fwhm). The fundamental beam of the wavelength $1.06 \mu \mathrm{m}$ was used for generation of a picosecond continuum in isopropanol, where the white light obtained was strong enough for absorption measurements within the wavelengths interval from $340 \mathrm{~nm}$ to $850 \mathrm{~nm}$. This broad continuum band was directed to the sample through a prism delay line built on the basis of a linear translation stage M-525.22 (Physik Instrumente) of the travel length $200 \mathrm{~mm}$, resolution $0.16 \mu \mathrm{m}$ and repeatability $0.4 \mu \mathrm{m}$, which ensured the maximum delay of $1.2 \mathrm{~ns}$ in steps of a few fs. The position of the zero time scale was established by using a fixed delay line placed in the excitation channel. The exciting and the analysing beams were arranged to cross at a small angle in the studied sample. Then, the exciting beam was blocked and the analysing one was directed through a low-dispersion spectrometer onto a 1453 silicone photodiode array (PDA) of an optical multichannel analyser (OMA), EG\&G/Princeton Applied Research, model 1460. The studied sample and the reference sample (usually of pure solvent) were placed on an additional translation stage which positioned them alternatively at the point of intersection of the exciting and the analysing beams. This permitted elimination of the background and all other reproducible disturbances originated in the system. The operation of the whole setup including the positioning of the 

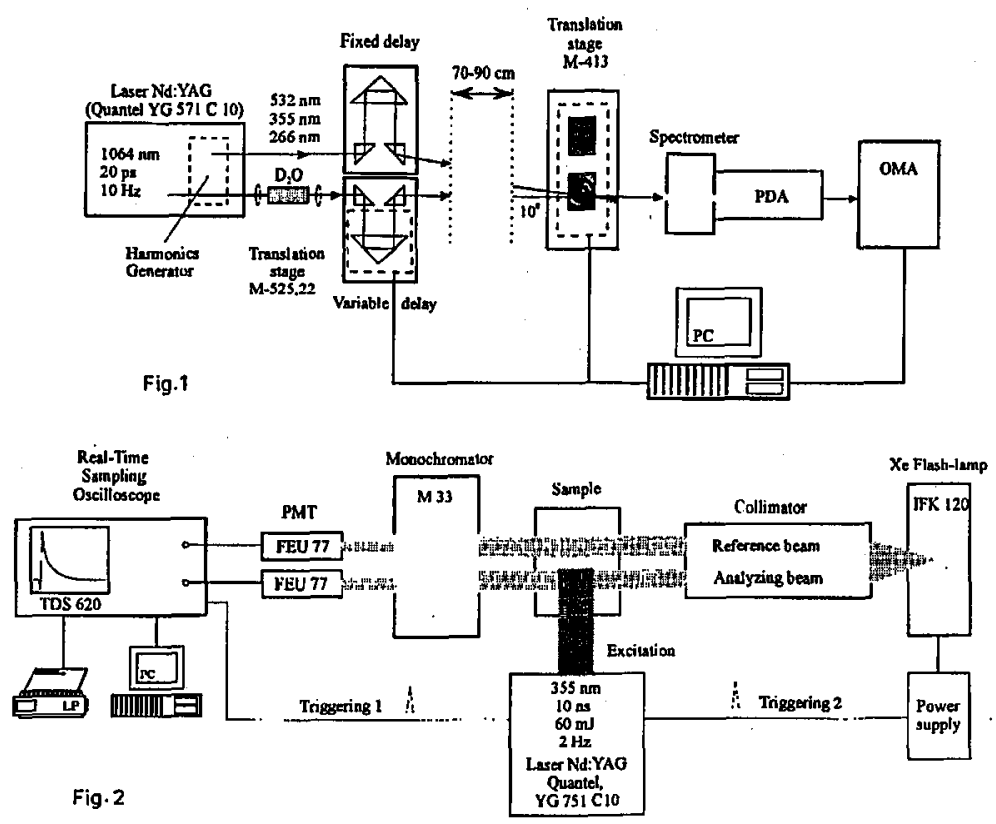

Fig. 1. A diagram of the experimental system for measurement of transient absorption spectra in the picosecond scale.

Fig. 2 A scheme of the nano/microsecond transient absorption spectrometer.

prism in the delay line, alternative exchange of the samples, synchronization with the laser, data acquisition, averaging, processing and display, was controlled by a PC 286 microcomputer. A more detailed description of the setup, its operation and experimental possibilities are given in Ref. [21].

Measurements of transient absorption in the nano- and microsecond time scales were carried out in a separate experimental setup, in which two parallel light beams from a pulse xenon lamp of flash duration $\approx 60 \mu$ s were directed onto two areas in the sample, one of them synchronously excited by the third harmonic $(355 \mathrm{~nm})$ of the fundamental beam from a YAG:Nd ${ }^{3+}$ laser. The same YG $571 \mathrm{C}$ (Quantel) laser which was used in the picosecond system was employed for generation of pulses of $10 \mathrm{~ns}$ duration (fwhm) and energy up to $90 \mathrm{~mJ}$ at a repetition rate of $2 \mathrm{~Hz}$. Transient absorption was measured in the real time with the use of two pulse FEU 77 photomultipliers. One of them measured the intensity of the part of the analysing beam which was passed through the non-excited region in the studied sample while the other measured the intensity of that part of the analysing beam which was passed through the laser excited region. The two parts of the analysing beam were mechanically separated but as they originated from the same flash of the lamp, they bore the same dependence of the light intensity. This way of measurement with two beams employed permitted differential processing of the transient absorption signal relative to the reference signal, which enhanced the sensitivity of the method and ensured that the results were independent of fluctu- 
ations of the analysing lamp beam intensity. A scheme of the nano/microsecond system for transient absorption measurements is shown in Fig. 2. The transient absorption and the reference signals were recorded in the real time using a Tektronix TDS 620 digital oscilloscope of a maximum sampling rate 2 Gs. After a preliminary treatment, i.e. generation of the differential signal and its averaging, the data were sent to a PC 286 microcomputer for further processing and storage. The same experimental setup was also used for investigation of luminescence decay in the nano- and microsecond range, performed with the analysing beam blocked and the emission signal only recorded. Absorption spectra were taken on a M-40 (Carl Zeiss Jena) spectrophotometer. Steady-state emission and excitation spectra were taken on an upgraded MPF-3 (Perkin-Elmer) spectrofluorimeter. The introduced modifications enabled single-photon counting detection, computer control and data processing achieved thanks to the application of a dual photon counting set (Light Scan). The spectrometer was also equipped with a reference quantum counter measuring intensity of the incident beam directly in front of the sample.

Quantum yields of emission were measured by the relative method, taking into account appropriate solution refractive index corrections [22, 23]. 9,10-diphenylanthracene in cyclohexane, $\phi_{\mathrm{F}}=0.91$ [22], and quinine sulfate in $0.1 \mathrm{~N}$ $\mathrm{H}_{2} \mathrm{SO}_{4}, \phi_{\mathrm{F}}=0.52$ [24], were used as standards. For quantum yield and corrected excitation spectral measurements the optical density of the samples was always $<0.05$ and the same spectral bandpasses were used for both absorption and emission experiments.

The thiones used in our experiments were synthesized by reaction of the corresponding ketone (Aldrich) with $\mathrm{P}_{4} \mathrm{~S}_{10}$ following the methods of Arndt and Lorenz [25], Pedersen et al. [26] and Scheermer et al. [27]. The crude products were recrystallized several times from ethanol and toluene and stored in the dark at a low temperature in the absence of air. Purities were monitored by UV spectrophotometry with the use of a Carl Zeiss model M-40 instrument and by gas chromatography using a Hewlett-Packard model 5880A instrument fitted with a $50 \mathrm{~m}$ SE-52 capillary column. Perfluoroalkanes (PCR Research Chemicals) as well as aliphatic and aromatic hydrocarbon solvents (Aldrich) were purified by column chromatography and fractional distillation. Thione solutions. were deoxygenated by bubbling $\mathrm{O}_{2}$-free $\mathrm{He}$ through them.

\section{Results}

Spectral and photophysical properties of solutions of a few representative aromatic thioketones in benzene, alkanes and perfluoroalkanes were studied. These properties of the studied thioketones: xanthione, XT; 4H-1-benzopyran-4-thione, BPT; 2,2,3,3-tetramethylindanethione, TMIT; 4-bromo-2,2,3,3-tetramethylindane-thione, Br-TMIT; whose structural formulae are given in Fig. 3, are very close $[10,28]$ and reveal mostly only quantitative differences.

For our studies we have chosen XT and BPT as typical representatives of rigid aromatic thiones, as well as TMIT and BrTMIT in order to check whether the presence of bromine atom in the latter (the only difference between these two compounds) would affect their photophysical properties and deactivation pathways from the $S_{2^{-}}$and $T_{1}$-states. The energy difference between these states 
<smiles>S=c1c2ccccc2oc2ccccc12</smiles>

$\mathrm{XT}$<smiles>CC1(C)Cc2ccccc2C1=S</smiles>

TMlT<smiles>S=C1CCOc2ccccc21</smiles>

BPT<smiles>CC1(C)C(=S)c2cccc(Br)c2C1(C)CBr</smiles>

Fig. 3. Structural formulae of the studied thioketone molecules.

TABLE I Quantum yields and $S_{2}$-fluorescence decay times determined for chosen aromatic thioketones in solutions with perfluoro-1,3-dimethylcyclohexane, PFDMCH, 3-methylpentane, $3 \mathrm{MP}$, and benzene, $\mathrm{C}_{6} \mathrm{H}_{6}$, at a concentration $[c] \approx 10^{-5} \mathrm{M}$ at $\mathrm{RT}$. $E_{S_{2}}$ denotes the energy of $S_{2}$-state determined in PFDMCH.

\begin{tabular}{|c|c|c|c|c|c|c|c|}
\hline \multirow[b]{2}{*}{ Thione } & \multirow{2}{*}{$\begin{array}{c}E_{S_{2}} \\
{[\mathrm{kcal} / \mathrm{mol}]}\end{array}$} & \multicolumn{3}{|c|}{$\phi_{\mathrm{F}}\left[\times 10^{-2}\right]$} & \multicolumn{3}{|c|}{$\tau_{\mathrm{F}}[\mathrm{ps}]$} \\
\hline & & PFDMCH & $3 \mathrm{MP}$ & $\mathrm{C}_{6} \mathrm{H}_{6}$ & PFDMCH & $3 \mathrm{MP}$ & $\mathrm{C}_{6} \mathrm{H}_{6}$ \\
\hline $\mathrm{XT}$ & 68.3 & $\begin{array}{c}1.3 \\
1.4[2]\end{array}$ & 0.19 & 0.18 & $178[7]$ & $25[17]$ & 11 [17] \\
\hline $\mathrm{BPT}$ & 72.8 & $\begin{array}{c}1.7 \\
2.3[2]\end{array}$ & 0.18 & 0.15 & $210[7]$ & $25[7]$ & $13^{a}$ \\
\hline TMIT & 83 & $\begin{array}{c}9.5 \\
14[2]\end{array}$ & 1.2 & 0.36 & $880[2]$ & $77[7]$ & $22^{a}$ \\
\hline BrTMIT & 80.6 & 0.6 & 0.4 & 0.3 & 35 & 27 & $18^{a}$ \\
\hline
\end{tabular}

${ }^{a}$ Calculated from the Strickler-Berg formula [33, 34].

TABLE II

Quantum yields and $T_{1}$-phosphorescence decay times for chosen aromatic thioketones in solutions with perfluoro-1,3-dimethylcyclohexane, PFDMCH, 3-methylpentane, $3 \mathrm{MP}$, and benzene, $\mathrm{C}_{6} \mathrm{H}_{6}$, extrapolated to the zero concentration of the thione. $E_{T_{1}}$ denotes energy of $T_{1}$-state determined in PFDMCH.

\begin{tabular}{l|c|c|c|c|c|c|c}
\hline \hline \multirow{2}{*}{ Thione } & $E_{T_{1}}$ & \multicolumn{3}{|c|}{$\phi_{\mathrm{P}}^{0}\left[\times 10^{-2}\right]$} & \multicolumn{4}{c}{$\tau_{\mathrm{P}}^{0}[\mu \mathrm{s}]$} \\
\cline { 3 - 8 } & {$[\mathrm{kcal} / \mathrm{mol}]$} & $\mathrm{PFDMCH}$ & $3 \mathrm{MP}$ & $\mathrm{C}_{6} \mathrm{H}_{6}$ & PFDMCH & $3 \mathrm{MP}$ & $\mathrm{C}_{6} \mathrm{H}_{6}$ \\
\hline $\mathrm{XT}$ & 43.2 & 3.0 & 2.7 & 1.0 & $8.2[5]$ & $7[4]$ & 2.5 \\
BPT & 45.8 & 7.0 & 3.0 & 1.4 & $16[5]$ & $7.3[4]$ & 3.3 \\
TMIT & 45.6 & 6.3 & 6.4 & 2.1 & 38 & 30 & 7.5 \\
BrTMIT & 44.4 & 7.0 & 4.2 & 2.3 & 22 & 18 & -
\end{tabular}


is significant, $\approx 35 \mathrm{kcal} / \mathrm{mol}$, which may have essential influence on the value of the rate constant of bromine atom abstraction from BrTMIT and thus, on the $\tau_{\mathrm{F}}$ and $\tau_{\mathbf{P}}$ lifetimes. As solvents we used: perfluoro-1,3-dimethylcyclohexane, PFDMCH, 3-methylpentane, 3MP, and benzene, $\mathrm{C}_{6} \mathrm{H}_{6}$. As we have shown many times [2-10,29], only the use of perfluoroalkanes as solvents ensures the possibility of investigating intramolecular properties of thiones in the $S_{2}$ - and $T_{1}$-states. The use of 3MP, a typical aliphatic hydrocarbone, ensures that the interactions with thioketones in the $S_{2}$ and $T_{1}$-state are limited to the reaction of hydrogen abstraction only $[5,7-10,15,16]$ as the universal (physical) interactions are in it negligibly weak $[5,8-10,29,30]$. The third of the applied solvents, benzene, is highly chemically inert, so the reaction of hydrogen abstraction from its molecules practically does not occur, which means that the photochemical processes are eliminated from the deactivation of the $S_{2}$ - and $T_{1}$-state thiones. In this way, the use of benzene permits investigation of physical and $\pi$-electron (quantum mechanical type) interactions with thione molecules. This should lead to highly efficient deactivation of the $S_{2}$-states $[7,10,17,18]$ and to formation of excited complexes (exciplexes) which are untypical in the sense that they are formed in the $S_{2}$-state, which provides a possibility of their deactivation to lower, bonding exciplex states. Moreover, the results obtained with benzene used as solvent can be compared with the data from earlier studies of transient absorption in thiones performed in the picosecond $[18,20]$, nanosecond and microsecond $[14,31,32]$ scale in benzene.

Tables I and II present results of measurements and relevant literature data on quantum yield of fluorescence and phosphorescence, together with the corresponding decay times, for the above mentioned thioketones in three chosen solvents. The values in Table II are extrapolated to zero concentration of thioketones in solutions, so they have been determined from Stern-Volmer equations on the basis of the measurements performed as a function of thioketone concentration. As follows from Table I, the quantum yield of fluorescence and the $S_{2}$-state lifetimes are much smaller for thiones in 3MP than in PFDMCH. Still smaller values of $\phi_{\mathrm{F}}$ and $\tau_{\mathrm{F}}$ have been obtained in $\mathrm{C}_{6} \mathrm{H}_{6}$. The effect of the solvent is the greater the higher are the values of quantum yield or lifetime measured in perfluoroalkane.. A similar effect has been observed for quantum yield of phosphorescence and lifetimes of the $T_{1}$-state, illustrated in Table II, although on a much smaller scale.

Tables III and IV give the values of rate constants $k_{\mathrm{nr}}$ of radiationless decay of $S_{2^{-}}$and $T_{1}$-states and the determined values of fractions $F_{\text {inter }}$ describing the contribution of the intermolecular channel in the decay of appropriate states.

Such a significant solvent effect as indicated by Table I data has been rarely observed in typical photophysical systems since alkanes, and 3MP in particular, have been usually considered as unreactive and physically inert [35]. A comparison of quantitative as well as qualitative spectral, physical and chemical characteristics of PF and alkane solvents gives no obvious reasons for so profound differences in the thiones interaction with them (for a review, see Ref. [36]). Even the typically spectral properties of thiones in the two groups of solvents look alike. The energies of excited states, positions of the maxima in absorption and emission bands, shapes of the spectral bands, Stokes shifts as well as the intensities of the absorption bands expressed by a molar extinction coefficient value are similar $[4,5,7,10,29]$. 


\section{TABLE III}

The influence of a solvent on the rate constant of $S_{2}$-state nonradiative decay, $k_{\mathrm{nr}}$, and fraction of $S_{2}$ states decaying by intermolecular interactions; $F_{\text {inter }}^{S_{2}}$, for selected thiones.

\begin{tabular}{l|c|c|c|c|c|c}
\hline \hline \multirow{2}{*}{ Thione } & \multicolumn{3}{|c|}{$k_{\mathrm{nr}}^{a}\left[\times 10^{9} \mathrm{~s}^{-1}\right]$} & \multicolumn{3}{c}{$F_{\text {inter }}^{S_{2}}$} \\
\cline { 2 - 7 } $\mathrm{PFDMCH}$ & $3 \mathrm{MP}$ & $\mathrm{C}_{6} \mathrm{H}_{6}$ & PFDMCH & $3 \mathrm{MP}$ & $\mathrm{C}_{6} \mathrm{H}_{6}$ \\
\hline $\mathrm{BPT}$ & 5.5 & 39.9 & 90.7 & $0^{c}$ & 0.86 & 0.94 \\
TMIT & 4.7 & 39.9 & 76.8 & 0 & 0.88 & 0.94 \\
BrTMIT & 1.0 & 12.8 & 45.3 & 0 & 0.92 & 0.98 \\
\hline${ }^{a}$ Calculated from $k_{\mathrm{nr}}=\left(1-\phi_{\mathrm{F}}\right) / \tau_{\mathrm{F}}$. & 0 & 0.23 & 0.49 \\
${ }^{b}$ Calculated from $F_{\text {inter }}^{S_{2}}=1-\tau_{\mathrm{F}}(3 \mathrm{MP}) / \tau_{\mathrm{F}}(\mathrm{PFDMCH}), \tau_{\mathrm{F}}(3 \mathrm{MP})$ \\
and $\tau_{\mathrm{F}}(\mathrm{PFDMCH})$ denote the fluorescence decay times deter- \\
mined in PFDMCH and $3 \mathrm{MP}$, respectively. \\
$c$ Values of $F_{\text {inter }}^{S_{2}}$ in PFDMCH assumed to be zero.
\end{tabular}

TABLE IV

The influence of a solvent on the rate constant of $T_{1}$-state nonradiative decay, $k_{\mathrm{n}}$, fraction of $T_{1}$-states decaying by intermolecular interactions, $F_{\text {inter }}^{T_{1}}$, and the efficiency of $T_{1}$-state formation under $S_{2}$-state excitation, $\phi_{S_{2}, T_{1}}$, for a few thiones.

\begin{tabular}{|c|c|c|c|c|c|c|c|c|c|}
\hline \multirow[b]{2}{*}{ Thione } & \multicolumn{3}{|c|}{$k_{\mathrm{nr}}^{a}\left[\times 10^{5} \mathrm{~s}^{-1}\right]$} & \multicolumn{3}{|c|}{$\phi_{S_{2}, T-1}^{b}$} & \multicolumn{3}{|c|}{$F_{\text {inter }}^{T_{1}}$} \\
\hline & $\begin{array}{l}\text { PF- } \\
\text { DMCH }\end{array}$ & $3 \mathrm{MP}$ & $\mathrm{C}_{6} \mathrm{H}_{6}$ & $\begin{array}{l}\text { PF- } \\
\text { DMCH } \\
\end{array}$ & $3 \mathrm{MP}$ & $\mathrm{C}_{6} \mathrm{H}_{6}$ & $\begin{array}{l}\text { PF- } \\
\text { DMCH }\end{array}$ & $3 \mathrm{MP}$ & $\mathrm{C}_{6} \mathrm{H}_{6}$ \\
\hline $\mathrm{XT}$ & 1.1 & 1.3 & 3.9 & $0.61[13]$ & $0.65[13]$ & 0.6 & $0^{d}$ & 0.15 & 0.72 \\
\hline BPT & 0.54 & 1.3 & 3.0 & $0.58[13]$ & $0.59[13]$ & 0.65 & 0 & 0.58 & 0.82 \\
\hline TMIT & 0.23 & 0.29 & 1.3 & $0.57[13]$ & $0.53[13]$ & 0.7 & 0 & 0.21 & 0.82 \\
\hline BrTMTT & 0.4 & 0.49 & - & 0.5 & - & - & 0 & 0.18 & - \\
\hline
\end{tabular}

a Calculated from $k_{\mathrm{nr}}=\left(1-\phi_{\mathrm{P}}^{S_{2}} / \phi_{S_{2}, T_{1}}\right) / \tau_{\mathrm{P}}^{0}$.

${ }^{b} \phi_{S_{2}, T_{1}}=\phi_{\mathrm{P}}^{S_{2}} / \phi_{\mathrm{P}}^{T_{1}}, \phi_{\mathrm{P}}^{S_{2}}$ and $\phi_{\mathrm{P}}^{T_{1}}$ denote phosphorescence quantum yields measured under selective excitation of $S_{2}$ - and $T_{1}$-states, respectively.

$c^{c}$ Calculated from $F_{\text {inter }}^{T_{1}}=1-\tau_{\mathrm{p}}(3 \mathrm{MP}) / \tau_{\mathrm{P}}(\mathrm{PFDMCH}), \tau_{\mathrm{P}}(3 \mathrm{MP})$ and $\tau_{\mathrm{P}}(\mathrm{PFDMCH})$ denote phosphorescence decay times determined in 3MP and PFDMCH, respectively.

${ }^{d}$ Values of $F_{\text {inter }}^{T_{1}}$ in perfluoroalkane solvents assumed to be zero.

This similarity is also evident from Fig. 4, which shows quantitative absorption and emission spectra of a typical thioketone in alkane and perfluoroalkane. In contrast to the emission spectra reflecting the same solvent effect as manifested by the data from Tables I and II, the absorption spectra are practically the same. It should be emphasized that the absorption spectra of thioketones in a solvent are strongly dependent on the solute-solvent interactions and only in non-interacting perfluoroalkanes the shape of these spectra is close to those in noble gas matrices [36]. Thus, the similarity of the absorption spectra of tioketones in PFDMCH and 3MP testifies to the inertness and unreactivity of alkanes towards thioketones in the ground state. The absorption spectra of thioketones in benzene are 


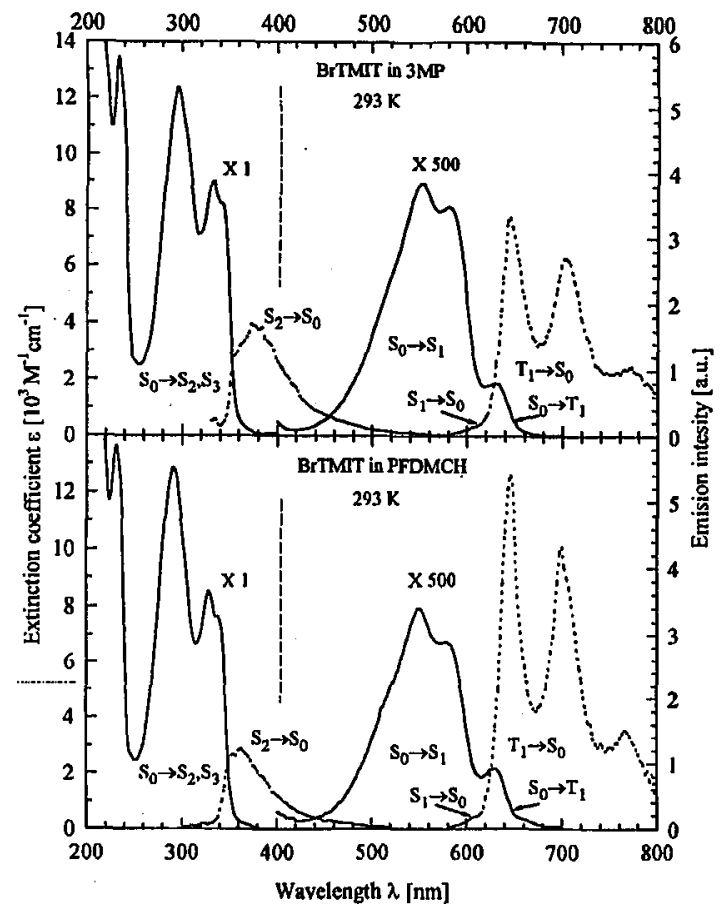

Fig. 4. Absorption and emission spectra of BrTMIT in an alkane (3-methylpentane, $3 \mathrm{MP}$ ) and in a perfluoroalkane (perfluoro-1,3-dimethylcyclohexane, PFDMCH). The $S_{0} \rightarrow S_{1}, T_{1}$ absorption spectra were measured at the solute concentration $[c] \approx$ $5 \times 10^{-3} \mathrm{M}, S_{0} \rightarrow S_{2}, S_{3}$ at $[c] \approx 1 \times 10^{-4} \mathrm{M}$, while the emission ones at $[c]=1.3 \times 10^{-5} \mathrm{M}$.

significantly different from those measured in PF and in alkanes. The differences are: a change in the shape of bands in the visible range and a lack of a separate $S_{0} \rightarrow T_{1}\left(n, \pi^{*}\right)$ absorption band, and they are interpreted as a result of universal (physical) interactions.

In order to detect a possible participation of the excited state individua, formed as a result of interaction with the solvent, in the decay of the $S_{2}$ - and $T_{1}$-states of thioketones, the transient absorption spectra of xanthione in the picoand nanosecond range have been examined. Figure 5 illustrates the time evolution of the transient absorption curves for a few chosen wavelengths. These dependences were measured, using the earlier described transient absorption spectrometer, for xanthione solution in benzene. The same data for the same system but in a form of a quasi-three-dimensional transient absorption spectrum are shown in Fig. 6a and $b$. Both parts of the figure present the same surface corresponding to the initial stages of the spectrum evolution, before the transient absorption signal gets stabilized, viewed at two different angles. The zero point on the time scale, that is the moment the exciting pulse started to act on the sample, was determined from the $T_{1}-T_{n}$ transient absorption measurements for benzophenone in $n$-heptane, reported in Ref. [21]. The same reference also gives a description of the way the 


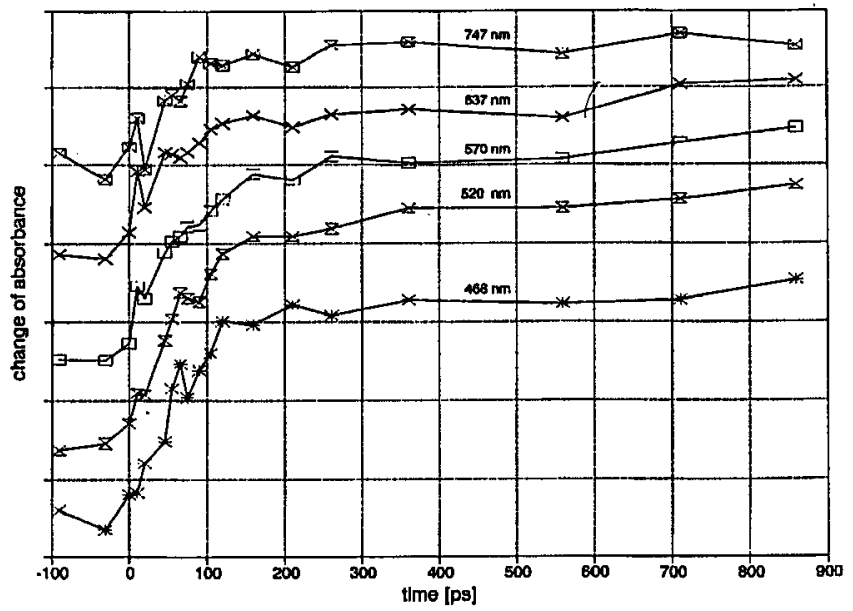

Fig. 5. The time dependence of transient absorption of xanthione in benzene for selected wavelengths. The absorbance scale unit is 0.1 . For the sake of clarity the curves have been vertically shifted by the absorbance unit.
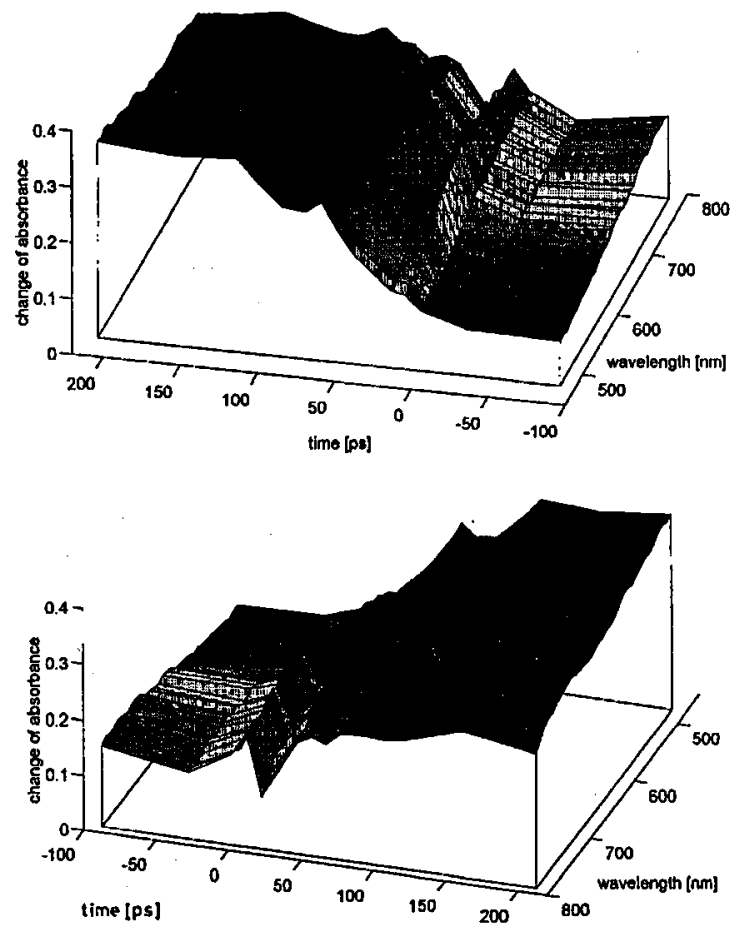

Fig. 6. The quasi-three-dimensional surface of transient absorption spectrum of XT in benzene viewed from two different angles. 


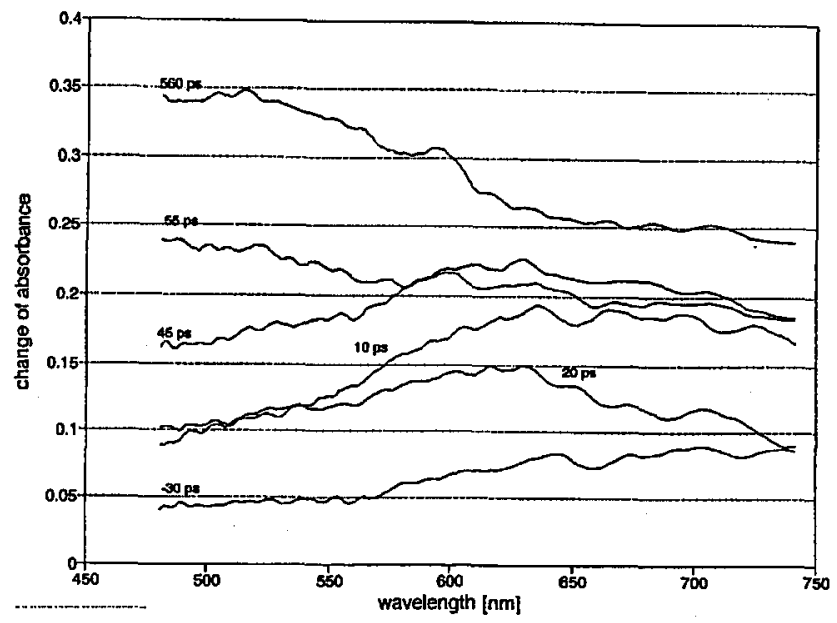

Fig. 7. Transient absorption spectra of xanthione in benzene measured for different times after excitation.

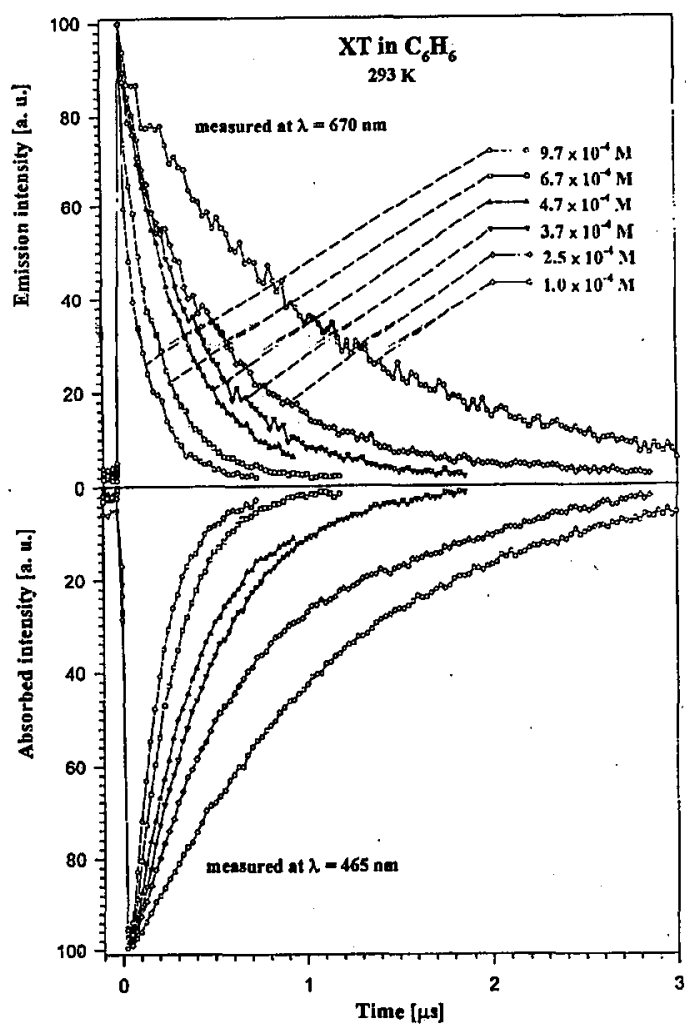

Fig. 8. Phosphorescence and transient absorption decay curves measured for xanthione in benzene solution. 
instrument response profile is determined by the two-photon fluorescence technique. Figure 7 presents the transient absorption spectra at particular moments obtained on the basis of the same data, i.e. the appropriate cross-sections of the surface from Fig. 6. As follows from Fig. 5, the transient absorption of XT does not disappear in the first nanosecond, which is the time window of the picosecond. spectrometer but it gets stabilized at an almost constant level. Further evolution of this signal studied by a nano/microsecond spectrometer and shown in Fig. 8, implies that the decay of the absorption marked in Fig. 5 takes place in the time scale of some microseconds.

The analysis of the transient absorption from Figs. 5, 6 and 7 provides important information about photophysical properties of the system thioketone/solvent, despite the noise and instabilities seen in the figures resulting from the complex technique of measurement. The curves of transient absorption evolution from Fig. 5 on the left of the zero point illustrate the sample absorption prior to the exciting pulse. Upon the sample excitation, a peak in transient absorption with a maximum in the red part of the spectrum appears. It is sharp in the time scale (Fig. 5) but broad in the wavelength scale (Figs. 6, 7). A characteristic feature of the peak is its synchronous appearance in all curves from Fig. 5 recorded in the long-wave part of the spectrum. This peak has been ascribed to the $S_{2} \rightarrow S_{n}$ absorption realizing that as it appears in the first picoseconds after excitation it must be due to the directly excited individuum (singlet XT), and the time width of this peak corresponds to the lifetime of XT in the $S_{2}$ state (11 ps [17]) convoluted with the width of the exciting pulse. Moreover, the spectrum of this absorption corresponds to the $S_{2} \rightarrow S_{n}$ absorption recorded by Hochstrasser et al. [18]. The above transient absorption peak shows a fast decay and after passing through a minimum at about $50 \mathrm{ps}$ the absorption signal begins to grow, at first rapidly (with a few fluctuations we shall disregard at the moment) and then at a slower rate. The phase of the signal increase lasts for about $150 \mathrm{ps}$ and the maximum of the signal is now shifted to the blue (Figs. 6,7). We shall leave the increase phase of the long time absorption for further discussion in Sec. 4 and now we shall concentrate on the nature of the already stabilized signal of transient absorption at the end of the time scale in Fig. 5. In order to perceive the signal in its specific time scale one should analyse it in view of Figs. 8 and 9 which present the time and spectral evolution of this "slow" transient absorption in the microsecond scale for solutions of different concentration of XT. The upper part of Fig. 8 presents the curves of phosphorescence decay from the $T_{1}$-state of xanthione determined for the same samples which were used in the absorption measurements. There is strong evidence pointing to the $T_{1} \rightarrow T_{n}$ transitions as mainly responsible for the long time transient absorption. First of all the decay time of this absorption is equal to the $T_{1}$-state lifetime, which can be inferred from Fig. 8 showing clearly that the phosphorescence decay and transient absorption decay run at almost the same rate constants. Secondly, the effect of selfquenching on both processes is the same and the rate constant of this process corresponds to the diffusion-controlled selfquenching of the $T_{1}$-state. Moreover, the transient absorption spectrum shown in Fig. 9 is very similar to the one reported by Das et al. [31]. To sum up, taking into regard the properties of the studied system, it would be difficult to suggest an 


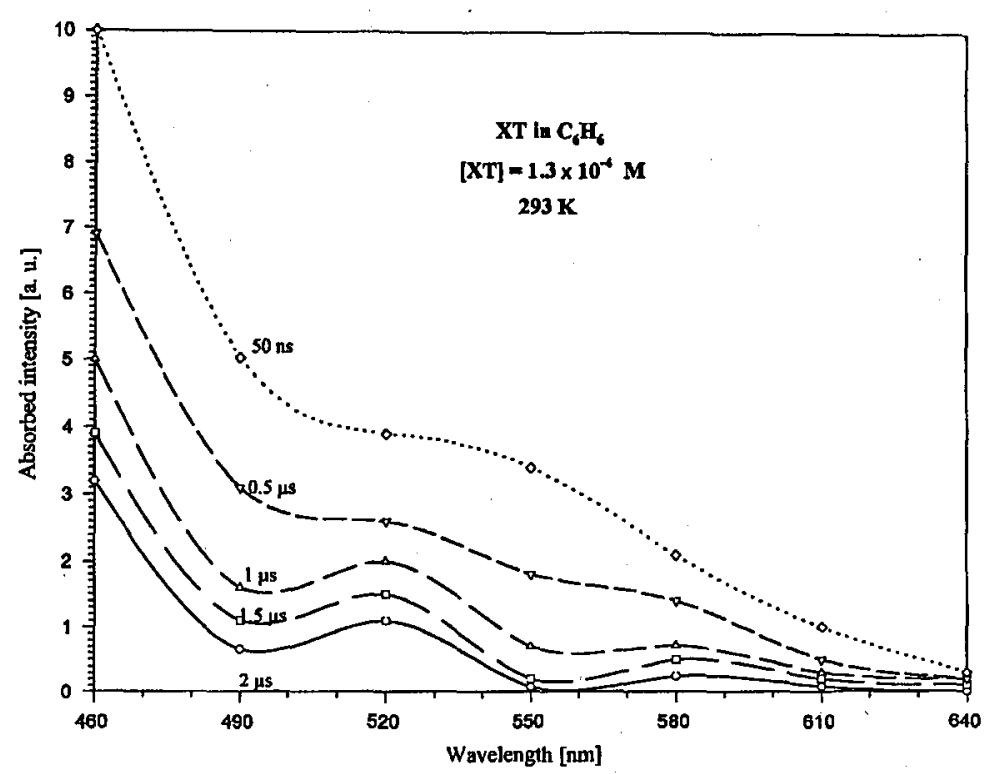

Fig. 9. The transient absorption spectra of xanthione in benzene solution measured for various times after the excitation pulse.

alternative origin of this absorption which is both in spectral and time distribution different from the absorption occurring directly after excitation.

\section{Discussion}

The evidence on the photophysical properties of thioketones reported in this and previous papers as well as by other authors reveals drastic differences between the thioketone behaviour in perfluoroalkane, alkane and benzene solutions. As follows from Tables I-IV, an alkane and even more benzene used as solvents, act as effective quenchers of excited states and the differences in the solvent effect are particularly pronounced for the thiones excited to the $S_{2}$-state although the same effect has been shown for the $T_{1}$-state. Of course, the effect of the solvent is the greater the longer the lifetime of a particular state of a given thione as then the contribution of the quenching through the interaction with the solvent in the total deactivation, increases. The dependence of the solvent effect magnitude on the lifetime of an electronic level is particularly well seen when the analogous states of different thiones described in Tables I-IV, are compared.

An interesting result is a significant (about 20 times) shortening of $\tau_{\mathrm{F}}$ and a decrease in $\phi_{F}$ in BrTMIT with respect to the values obtained in TMIT, Table I. This effect must be related to the presence of the $\mathrm{C}-\mathrm{Br}$ bond in a BrTMIT molecule and must be of intramolecular nature as it is observed in PF. In the case of the $T_{1}$-state, the $\tau_{\mathrm{P}}^{0}$ value in BrTMIT is less than twice smaller than its value in TMIT while $\phi_{\mathrm{P}}$ does not change at all. Since the energy of the $T_{1}$-state of thiones is relatively low, the potentially possible process of the $\mathrm{C}-\mathrm{Br}$ bond dissociation should not play an important role in the $T_{1}$-state deactivation. A close value of $\phi_{\mathrm{P}}^{0}$ 
for both thiones despite a significantly shorter $\tau_{\mathrm{P}}^{0}$ in BrTMIT indicates that the presence of $\mathrm{Br}$ induces a typical internal heavy atom effect which is responsible for an increase in the spin-orbit coupling and affects the radiative rate constant more than the rate constant of radiationless transition to the ground state [37].

As follows from Table III, the rate constant of radiationless deactivation of the $S_{2}$-state, takes the highest values for all thiones in $\mathrm{C}_{6} \mathrm{H}_{6}$ and for XT reaches $1 \times 10^{11} \mathrm{~s}^{-1}$. Therefore the intermolecular interactions leading to formation of exciplex in the $S_{2}$-state are responsible for deactivation of the studied thiones in over $90 \%$. The only exception is BrTMIT which in the $S_{2}$-state undergoes effective deactivation along an intramolecular path. Since the energies of the $S_{2}$-state, $E_{S_{2}}$, are high and of similar value for both TMIT and BrTMIT, it seems that such untypical behaviour of BrTMIT in the $S_{2}$-state may be interpreted as due to efficient dissociation of a relatively weak $\mathrm{C}-\mathrm{Br}$ bond leading to formation of radicals. A low quantum yield of photochemical decay of BrTMIT in these conditions $\phi_{\mathrm{PH}}<10^{-2}$, means that the process is reversible and so it should be an effective channel of radiationless deactivation of photochemical nature.

A high value of the fraction $F_{\text {inter }}^{S_{2}}$ given in Table III, indicating what part of the $S_{2}$ thione decay occurs via intermolecular interactions, testifies to a high yield of formation of the exciplex in the $S_{2}$-state by molecules of XT in the $S_{2}$-state and $\mathrm{C}_{6} \mathrm{H}_{6}$ in the $S_{0}$-state. It can be expected that in spite of the high yield of the above process, the energy of complex formation is low $[38,39]$ which follows from the nature of interactions leading to the complex formation [11, 40, 41]. Most probably these remarks apply also to the complex formed by XT in the $T_{1}$-state and $\mathrm{C}_{6} \mathrm{H}_{6}$ molecule in the $S_{0}$-state.

An interesting finding given in Table III are very high and similar values of $F_{\text {inter }}^{S_{2}}$ obtained in $3 \mathrm{MP}$ and $\mathrm{C}_{6} \mathrm{H}_{6}$ for all thiones except BrTMIT in which, as has been pointed out earlier, there is a competitive channel of intramolecular deactivation. It can be explained by the fact that reactivity of thiones in the $S_{2}$-state is so remarkably high that despite a completely different mechanism, the rates of quenching via intermolecular processes with the solvent are close in both cases.

It is also interesting to compare the rate constants of radiationless decay $k_{\mathrm{nr}}$ of thiones excited to the $T_{1}$-state, given in Table IV. When 3MP is used as solvent, its contribution in deactivation of thiones is small (except for BPT), which is indicated by a low value of $k_{\mathrm{nr}}$, close to that for thiones solved in PF. This is explained by a low probability of hydrogen abstraction from 3MP molecules in the endothermic process of interaction with thiones in the $T_{1}$-state of low energy, quite contrary than in the case of ketones [42]. In the benzene solutions $k_{\mathrm{nr}}$ is significantly greater than in PF (Table IV), and $F_{\text {inter }}^{T_{1}}$ is high as the process of interaction of the thiones in the $T_{1}$-state with $\mathrm{C}_{6} \mathrm{H}_{6}$ is the main channel of their deactivation.

As mentioned earlier, it is the formation of excited state individua breaking up into a molecule of thione in the $T_{1}$-state and a solvent molecule, that plays a crucial role in the decay of thioketones in their $S_{2}$-state in benzene or alkane solvents. This process is reversible so it does not give stable products and its photophysical result is the drastic solvent effect. The so far performed experi- 
mental attempts at detecting the emission of this unstable individuum, involving both steady-state and dynamic measurements, have failed which suggests that the searched individuum must be a dark species.

The time resolved transient absorption spectra measured in our experiment also do not reveal the transient absorption bands which could be ascribed directly to such individua. Nevertheless, in our opinion, these spectra confirm their presence and role in the $S_{2}$-thione decay. As shown in Sec. 3, the transient absorption spectrum of XT in $\mathrm{C}_{6} \mathrm{H}_{6}$ includes two main components: the $S_{2} \rightarrow S_{n}$ absorption of short duration and the slowly increasing $T_{1} \rightarrow T_{n}$ absorption of long duration which disappears in the scale of microseconds. The former band disappears in about 20 picoseconds as a direct consequence of a very short lifetime of xanthione in the $S_{2}$-state which in $\mathrm{C}_{6} \mathrm{H}_{6}$ is only $11 \mathrm{ps}$ [17]. After this state has decayed, all other singlet states of XT are also empty as they live shorter than the $S_{2}$-state. However, the population of the $T_{1}$-state still increases and this increase does not stop earlier than $150 \mathrm{ps}$ after the absorption from $S_{2}$-state disappeared. Therefore, as the increase in the $T_{1}$-state population takes so long, there must be an unstable $\left(\tau_{1 / \mathrm{e}} \geq 60 \mathrm{ps}\right)$ individuum in the system which would deactivate to the $T_{1}$-state of $\mathrm{XT}$, as it was suggested on the basis of steady-state measurements [5, 15].

A thorough analysis of the long time component of the picosecond transient absorption spectrum (Figs. 6,7) and the spectrum measured in the nanosecond transient absorption spectrometer (Fig. 9) reveals that the two spectra are not identical. The spectrum measured after $50 \mathrm{~ns}$ is certainly the pure spectrum of the $T_{1}-T_{n}$ transition as in this time all singlet states of XT as well as the exciplexes formed with $\mathrm{C}_{6} \mathrm{H}_{6}$ molecules have surely vanished and the admixture of the excimer in the $T_{1}$-state, which can be formed as a result of XT selfquenching in the $T_{1}$-state, is negligibly small. A different shape of the picosecond spectrum taken after a few liundred ps and the nanosecond one taken after 50 ns suggests that the former one must originate from more than one individuum. Taking into regard that the lifetime of this postulated individuum is of at least a few hundred ps, a probable candidate is the exciplex in the $S_{1}$-state formed via one of the two possible paths of $S_{2}$-exciplex decay.

There are a few probable reasons for making obscure the band which could be ascribed directly to the searched individuum. First of all the $S_{2} \rightarrow S_{n}$ and $T_{1} \rightarrow T_{n}$ transient absorption bands are so broad and shallow that it is difficult to identify the contributions of all components, particularly those of a low molar coefficient of extinction. Thus, it is impossible to perform a quantitative deconvolution of the "fast" absorption band in order to find out what are the contributions due to the $S_{2}$-state of XT and the excited states of the transient individuum. Moreover, the energy states of the transient individuum generated as a result of interaction of thioketone with the solvent, are because of this very interaction, localized on the energy scale lower than the singlet states of the thione, but the energy gaps between the corresponding states of the individuum and the singlet states of the thione should be similar. This means that the transient absorption spectra of the thione and the searched individuum can overlap. It is reasonable to expect that for the triplet states of the thione and the individuum the situation is similar. However, although the first phase of transient absorption from the triplet states 
does not indicate a participation of any triplet transient individuum, the time dependence of the spectra in the microsecond range reveals a characteristic feature at about $550 \mathrm{~nm}$ (Fig. 9), which must be due to the presence of a component other than the initial thione. It should be noted that this component is revealed with increasing time in the microsecond scale. This suggests the excimer nature of this individuum which on the one hand must have a long decay time and on the other its concentration should increase with the decay of the $T_{1}$-state of XT. These expectations are met by the $T_{1}$-excimer forming in selfquenching of XT in the $T_{1}$-state.

The results of our experiment allow us to infer about the physical nature of the transient individuum taking part in the decay of the $S_{2}$-state. Because of a low concentration of XT $\left(\approx 10^{-4} \mathrm{M}\right)$ and a very short lifetime of xanthione in the $S_{2}$-state in the XT/benzene system, the excimer character of this transient individuum can be excluded. On the other hand, great chemical inertness of benzene, which is a direct consequence of high energy of C-H bonds, prevents hydrogen abstraction from benzene molecule. These facts considered in view of a very efficient interaction between XT in the $S_{2}$-state and benzene in the ground state indicate that in this system the individuum intermediate in deactivation of the $S_{2}$-state and formation of the $T_{1}$-state must be a singlet exciplex.

Transient absorption spectra were also obtained for the systems XT/alkane (isooctane, hexane), however the situation in such systems is different. The interaction of thioketone in the $S_{2}$-state with alkane molecule in the ground state is also very efficient but most probably it involves hydrogen abstraction from the solvent molecule and formation of a thioketyl radical. This process is concluded to be responsible for a dramatic reduction of the $S_{2}$-state lifetime from $178 \mathrm{ps}$ for XT in PFDMCH to $35 \mathrm{ps}$ in isooctane and $25 \mathrm{ps}$ in $\mathrm{C}_{6} \mathrm{H}_{14}$. Such a reduction indicates that over $83 \%$ and $88 \%$ of XT molecules in $S_{2}$-state - in isooctane and hexane, respectively - deactivate as a consequence of the interaction with the solvent. As $\tau_{\mathrm{F}}$ determined for XT in isooctane and hexane is much longer than in benzene, the decay of the $S_{2}$-state occurs in parallel with the process of increasing $T_{1}-T_{n}$ absorption. During this increase in $T_{1}-T_{n}$ absorption we cannot distinguish the time interval in which the $S_{2}$-state of XT is practically empty so we cannot apply a simple description such as for the system XT/benzene and interpretation becomes much more complex.

The above conclusions concerning the physical nature of transient individua forming in different systems have been confirmed by results of the studies on the $\mathrm{H} / \mathrm{D}$ isotopic substitution effect in the solvent on the photophysical properties of thioketones. Deuteration of benzene practically affects neither the $S_{2}$-state lifetime nor the quantum yield of xanthione fluorescence. In $\mathrm{C}_{6} \mathrm{H}_{6}, \tau_{\mathrm{F}}$ is 11 ps $[15,17]$ or $12 \mathrm{ps}$ [18], whereas in $\mathrm{C}_{6} \mathrm{D}_{6}, \tau_{\mathrm{F}}=12 \mathrm{ps}[15,17]$. In both solvents $\phi_{\mathrm{F}}$ was determined to be $2 \times 10^{-3}$ [15]. The fact that $\tau_{\mathrm{F}}$ and $\phi_{\mathrm{F}}$ values do not change upon the $\mathrm{H} / \mathrm{D}$ isotopic substitution in the solvent is fully consistent with the conception of exciplex formation in this system. In the systems in which the interaction of excited thioketone with a molecule containing a $\mathrm{C}-\mathrm{H}$ bond leads to complete or partial hydrogen atom abstraction, the effect of the $H / D$ substitution should be significant. Indeed, for $\mathrm{XT}$ in $\mathrm{C}_{12} \mathrm{H}_{26} \tau_{\mathrm{F}}=22 \mathrm{ps}$ and $\phi_{\mathrm{F}}=2.6 \times 10^{-3}$, whereas in 
$\mathrm{C}_{12} \mathrm{D}_{26} \tau_{\mathrm{F}}=52 \mathrm{ps}$ and $\phi_{\mathrm{F}}=6.3 \times 10^{-3}$ [15]. The magnitude of the H/D isotopic effect is described by a factor of $c a .2 .4$ which is a value typical of the systems of this kind. These results imply an important role of hydrogen abstraction in quenching of the excited states of thioketones through interaction with alkane solvents which is also consistent with the idea of dark radical formation.

\section{Conclusions}

The above reported results of the studies of thioketone properties in solutions permitted a quantitative description of the effects of a very efficient interaction of excited thiones with 3-methylpentane as a typical representative of alkanes and benzene as a representative of aromatic hydrocarbons. For thioketones in the $S_{2}$-state the radiationless decay rate constant $k_{\mathrm{nr}}$, describing a combined effect of all intra- and intermolecular processes is in 3MP by an order of magnitude greater than in perfluoro-1,3-dimethylcyclohexane (which does not enter into intermolecular interactions) and in benzene even two or three times greater than in 3MP. Despite the essentially different mechanisms of interaction postulated for the two kinds of solvents (in 3MP the dominant is hydrogen abstraction process while in benzene - interaction of physical (universal) nature and different values of $k_{\mathrm{nr}}$, in both groups of active solvents due to the effectiveness of the relevant interactions, the fractions of thiones in the $S_{2}$-state which undergo decay via intermolecular path are almost the same and equal to about $90 \%$. Similar effects have been observed when thiones in the $T_{1}$-state interacted with representatives of the above mentioned groups of solvents although in this case, as the energy of the lowest triplet was lower, the scale of the solvent effect was smaller. In order to study the effect of the efficient intramolecular processes on the radiationless decay of thioketones in the $S_{2}$ - and $T_{1}$-states in solutions ensuring efficient excited solute-solvent interactions, we decided to study also BrTMIT whose molecular structure permits efficient abstraction of $\mathrm{Br}$ which acts as a heavy atom and thus induces the increased spin-orbit coupling. A comparison of photophysical properties and parameters of decay of BrTMIT and TMIT in the $S_{2^{-}}$and $T_{1}$-states in different kinds of solvents leads to a conclusion that the efficient intramolecular processes can significantly affect the decay of excited states even in solvents ensuring highly efficient intermolecular interactions.

Using the method of transient absorption spectroscopy for XT in benzene, isooctane and hexane, we have made an attempt to find an experimental proof for the occurrence of a transient individuum which would intermediate in the processes of decay and which in benzene should be an exciplex whereas in alkanes a thioketyl radical. Although in the recorded spectra of transient absorption no bands were found which would have been ascribed directly to the searched intermediate, the curve of transient absorption increase obtained for XT/benzene unambiguously testifies to a contribution of a certain transient individuum in the decay of XT in the $S_{2}$-state. As follows from the properties of the studied system, such an individuum can only be an exciplex in the $S_{2}$-state of the decay time $\geq 60$ ps. Similar results were obtained for XT in alkanes but their interpretation is very difficult because of simultaneous occurrence of the decay of transient absorption from the $S_{2}$-state and increase in absorption from the $T_{1}$-state. In the 
actually physically inert and chemically unreactive perfluoroalkanes [36], the excited molecules of thioketones decay via the intramolecular path, as well as in the range of high concentrations and for long-lived states via selfquenching.

The transient absorption spectra of XT in benzene, measured a few tens ns after excitation (Fig. 9), are in good agreement with the particular results of Das et al. [31] concerning the $T_{1}-T_{n}$ transient absorption in the long time limit. Moreover, the shapes of the absorption spectra for short times after excitation are consistent with the results reported by Anderson et al. [18].

The microsecond transient absorption spectra of XT reveal some changes increasing with progressing decay, which, on the basis of the system properties have been ascribed tentatively to a triplet excimer.

This work reports results of transient absorption measurements performed only for the xanthione characterized by a short decay time of the $S_{2}$-state, chosen especially to simplify interpretation of results. However, taking into regard the generally well-recognized similarities in the properties of thioketones, and the fact that differences among them are mainly of quantitative character, it is reasonable to expect that the course of the radiationless decay of thioketones in excited states via intermolecular path will be similar.

In concluding, in view of the obtained results of the steady-state and dynamic measurements, the participation of short-lived intermediates formed as a result of the interaction between thioketone in the $S_{2}$-state and benzene or hydrocarbon solvents, in deactivation of thiones, can be considered as experimentally proved. The presence of such individua and their role in deactivation of thiones excited to $S_{2}$-state have been recently independently confirmed in photochemical studies [43]. Further investigation of the individua as well as the long-time evolution of the triplet states generated during the decay of these species are under way.

\section{Acknowledgment}

The authors wish to thank Ms. Maria Fidecka, M.Sc., for careful preparation of the samples. The work has been supported by the grant 223379203 of the Committee for Scientific Research.

\section{References}

[1] M. Kasha, Disc. Faraday Soc. 9, 14 (1950).

[2] A. Maciejewski, A. Safarzadeh-Amiri, R.E. Verrall, R.P. Steer, Chem. Phys. 87, 295 (1984).

[3] S.R. Abrams, M. Green, R.P. Steer, M. Szymański, Chem. Phys. Lett. 139, 182 (1987).

[4] A. Maciejewski, M. Szymański, R.P. Steer, J. Phys. Chem. 92, 6939 (1988).

[5] M. Szymański, A. Maciejewski, R.P. Steer, Chem. Phys. 124, 143 (1988).

[6] M. Szymański, R.P. Steer, A. Maciejewski, Chem. Phys. Lett. 135, 243 (1987).

[7] A. Maciejewski, D.R. Demmer, D.R. James, A. Safarzadeh-Amiri, R.E. Verrall, R.P. Steer, J. Am. Chem. Soc. 107, 2831 (1985).

[8] A. Maciejewski, Chem. Phys. Lett. 164, 166 (1989).

[9] A. Maciejewski, R.P. Steer, J. Photochem. 24, 303 (1984). 
[10] A. Maciejewski, R.P. Steer, Chem. Rev. 93, 67 (1993).

[11] A. Maciejewski, J. Photochem. Photobiol. A 43, 303 (1988).

[12] J. Kozłowski, A. Maciejewski, M. Szymański, R.P. Steer, J. Chem. Soc. Faraday Trans. 88, 557 (1992).

[13] M. Szymański, A. Maciejewski, R.P. Steer, J. Phys. Chem. 92, 2485 (1988).

[14] K. Bhattacharyya, P.K. Das, V. Ramamurthy, V.P. Rao, J. Chem. Soc. Faraday Trans. 2 82, 135 (1986).

[15] J. Kozłowski, Ph.D. Thesis, UAM, Poznan 1995.

[16] V. Ramamurthy, Organic Photochemistry 7, 232 (1986).

[17] C.J. Ho, A.L. Motyka, M.R. Topp, Chem. Phys. Lett. 158, 59 (1989).

[18] R.W. Anderson, R.M. Hochstrasser, H.J. Pownall, Chem. Phys. Lett. 43, 224 (1976).

[19] R. Minto, A. Samanta, P.K. Das, J. Phys. Chem. 67, 967 (1989).

[20] J. Kamphuis, H.J.T. Bos, R.J. Visser, B.H. Huizer, C. Varma, J. Chem. Soc. Perkin Trans. II, 1867 (1986).

[21] M. Szymański, M. Balicki, M. Binkowski, J. Kubicki, E. Pawłowska, T. Wróżowa, A. Maciejewski, Opt. Appl. 25, 1 (1995).

[22] S. Meech, D. Phillips, J. Photochem. 23, 193 (1983).

[23] M.O. Eidiger, R.S. Moog, S.G. Boxer, M.P. Fayer, Chem. Phys. Lett. 88, 123 (1983).

[24] B. Gelerent, A. Findeisen, J.A. Poole, J. Chem. Soc. Faraday Trans. 2 70, 939 (1973).

[25] F. Arndt, L. Lorenz, Berichte 63, 3121 (1930).

[26] B.S. Pedersen, S. Scheibye, N.H. Nilsson, S.O. Lawesson, Bull. Soc. Chim. Belg. 87, 223 (1978).

[27] J.W. Scheermer, P.H.J. Ooms, R.J.F. Nivard, Synthesis, 149 (1973).

[28] V. Ramamurthy, R.P. Steer, Acc. Chem. Res. 21, 380 (1988).

[29] A. Maciejewski, M. Szymański, R.P. Steer, J. Phys. Chem. 90, 6314 (1986).

[30] A. Maciejewski, M. Szymański, R.P. Steer, Chem. Phys. Lett. 143, 559 (1988).

[31] Ch.V. Kumar, L. Qin, P.K. Das, J. Chem. Soc. Faraday Trans. 2 80, 783 (1984).

[32] K. Bhattacharyya, V. Ramamurthy, P.K. Das, J. Phys. Chem. 91, 5626 (1987).

[33] S.J. Strickler, R.A. Berg, J. Chem. Phys. 37, 814 (1968).

[34] J.B. Birks, J. Res. Natl. Bur. Stand. A 80, 389 (1976).

[35] P. de Mayo, Acc. Chem. Res. 9, 52 (1976).

[36] A. Maciejewski, J. Photochem. Photobiol. 51, 87 (1990).

[37] S.P. McGlynn, T. Azumi, M. Kinoshita, Molecular Spectroscopy of the Triplet State, Prentice-Hall, Englewood Cliffs, New Jersey 1969.

[38] J. Dresner, J. Prochorow, W. Ode, J. Phys. Chem. 93, 671 (1989).

[39] I. Deperasińska, J. Prochorow, Chem. Phys. 114, 251 (1987).

[40] K. Morokuma, Acc. Chem. Res. 10, 294 (1977).

[41] H. Beens, A. Weller, in: Organic Molecular Photophysics, Ed. J.B. Birks, Vol. 2, Wiley, London 1975, p. 159.

[42] N.J. Turro, Modern Molecular Photochemistry, Benjamin and Cummings, New York 1978. 
[43] J. Kozłowski, A. Maciejewski, M. Milewski, M. Fidecka, in: Symposium on Physical Organic Photochemistry, Poznań 1995, Book of Abstracts, IIP-32, A. Mickiewicz University, Poznan 1995. 